\title{
Prestack reverse-time depth migration of arbitrarily wide-angle wave equations*
}

\author{
HE Bing-shou ${ }^{*}$ (何兵寿) ZHANG Hui-xing (张会星) ZHANG Jing (张 晶) \\ Marine Geo-science College, Ocean University of China, Qingdao 266100, China
}

\begin{abstract}
Based on arbitrarily wide-angle wave equations, a reverse-time propagation scheme is developed by substituting the partial derivatives of depth and time with central differences. The partial derivative of horizontal direction is replaced with high order difference. The imaging condition is computed by solving the eikonal equations. On the basis of above techniques, a prestack reverse-time depth migration algorithm is developed. The processing examples of synthetic data show that the method can remove unwanted internal reflections and decrease the migration noise. The method also has the advantage of fidelity and is applicable of dip angle reflector imaging.
\end{abstract}

Key words: acoustic waves; arbitrarily wide-angle wave equations; prestack reverse-time depth migration; absorbing boundary conditions; imaging conditions

CLC number: P $315.3^{+} 1 \quad$ Document code: A

\section{Introduction}

Prestack depth migration is a useful technique in seismic data processing of complex geological structure area. In the last years, the migration technique based on ray-trace theory played an important role in the seismic industry, but in recent years, with the increasing requirement for exploration precision and the increasing complexion of exploration target such as geometrical structure and lithology, the migration algorithms based on seismic wave equations are gradually adopted to solve seismic field imaging problems, which can improve ability of true amplitude processing. Geophysicist has achieved many important progress in wave equation prestack depth migration field, such as finite different migration algorithms of one-way wave equations (Claerbout, 1985), frequency-wave number migration algorithms (Gazdag, 1978) and reverse-time migration algorithms of two-way wave equations (Whitemore, 1983; Loewenthal et al, 1983; McMechan, 1983). In recent years, reverse-time migration algorithms aroused more and more attention owing to its advantages of high amplitude fidelity and the ability to solve dip angle structure imaging.

The idea of reverse-time migration was first presented by Whitemore et al (1983), then, Loewenthal and Mulfti (1983), McMechan (1983) and HE and ZHANG (2006) studied seismic wave reverse-time migration algorithms based on the two-way acoustic wave equation and the elastic wave equation, Symes (2007) studied a new migration method which can minimize computer storage at the price of a small increase in computation. All above research have greater

\footnotetext{
* Received 2007-12-06; accepted in revised form 2008-07-28.

Foundation item: China "863" Plan (2006AA06Z203).

*Author for correspondence: hebingshou@sina.com
} 
meaning for propelling the reverse-time migration technique forward. But until now, the successful cases using two-way wave-equation-based reverse-time migration in industry have been rarely seen, the main reasons for which are as follows: (1) in comparison with other migration algorithms, the reverse-time migration requires more computational efforts and internal computer memory; (2) there are still some technical problems that need to be further addressed for reverse-time migration, for example, suppressing numeric dispersion and pseudo-reflections of computing bounder; and (3) it has been difficult to overcome shortcomings in the theory of two-way wave-equation-based reverse-time migration. According to the two-way wave equation, when encountering an elastic interface in the process of reverse-time propagation, the seismic waves must produce up-going reflection waves that may not only suffer from loss of energy in the down-going waves, affecting the high fidelity of migrated results, but will also damage imaging in the shallow parts of section. This damage will result in the production of a lot of interference with strong amplitude and different frequencies, the band of which overlaps with that of useful waves and cannot be removed by mute in the frequency domain, resulting in a reduction in the imaging quality of the section.

Currently, along with rapid development of computer hardware and software technology, especially the successful application of micro-computer clusters in the field of seismic exploration, the aforementioned problem with computational efforts in reverse-time migration is no longer the bottleneck restraining large-scale industrial usage of this technique. The difficulty in suppressing the numeric dispersion and the pseudo-reflections from boundaries can be better solved by a small grid and PML absorption boundary (Berfenger, 1994). As for the third item in the above mentioned difficulties, there are two kinds of solutions; the first one is to modify the conventional two-way wave theory based migration algorithm. For example, Robin (2006) used the two-way wave equations in the whole computational space and one-way wave equation in special positions to reduce unwanted internal reflections, but this method need smoothing the interval velocity in vertical direction to remove the velocity discontinuity before migration, when an accurate velocity model is obtained, the smoothing of interval velocity is really a new contrived error; Yoon and Marfur (2006) used the Poyinting vectors to suppress the interval reflections of elastic interfaces but this can not produce satisfactory results in complex subsurfaces; Guitton et al (2007) improved the traditional filtering techniques and applying it to post-migrated data to filter the interval reflections, the nature of this method is a pre and post stack filter technique. Intrinsically speaking, the above-mentioned solutions are only the complement and perfection of two-way wave-equationbased reverse-time migration, and play a certain role in suppressing interval reflections, but cannot totally eliminate them. So, the two-way wave equation based on wave theory may certainly produce the reflections from an elastic interface. So long as the theoretical basis of the two-way wave equation is unchanged, the interference exists. The second kind of solution is to suppress the interval reflections abandoning the conventional two-way wave equation and use an appropriate one-way wave equation for implementation of reverse-time migration of the seismic wavefield. This paper uses an arbitrary wide-angle one-way acoustic wave equation to achieve the reverse-time migration of scalar seismic field. Guddati and Heidari (2005) deduced an arbitrary wide-angle one-way acoustic wave equation suitable for steep dip migration by reasonable simplification of the two-way acoustic wave equation. Using this equation, the paper studied the algorithm of the high-order finite-difference format for prestack reverse-time depth migration and its boundary, stability, and imaging conditions, and realized the prestack reverse-time depth migration of an arbitrary wide-angle wave equation in an isotropic medium. Through this they proved the effectiveness and accuracy of the method by migration processing of theoretical models. 


\section{Method and principle}

\subsection{Arbitrary wide-angle wave equation}

The arbitrary wide-angle wave equation deduced by Guddati and Heidari (2005) is:

$$
\boldsymbol{d} \frac{\partial^{2} u}{\partial z \partial t}+\frac{1}{c}\left(\boldsymbol{\Lambda}_{1}+\boldsymbol{\Lambda}_{2}\right) \frac{\partial^{2} \boldsymbol{u}}{\partial t^{2}}-c \boldsymbol{\Lambda}_{2} \frac{\partial^{2} \boldsymbol{u}}{\partial x^{2}}=0
$$

Where

$$
\begin{aligned}
& \Lambda_{1}=\frac{c}{2}\left[\begin{array}{cccccc}
\frac{1}{c_{1}} & -\frac{1}{c_{1}} & & & & \\
-\frac{1}{c_{1}} & \frac{1}{c_{1}}+\frac{1}{c_{2}} & -\frac{1}{c_{2}} & & & \\
& -\frac{1}{c_{2}} & \frac{1}{c_{2}}+\frac{1}{c_{3}} & -\frac{1}{c_{3}} & & \\
& & \ddots & \ddots & \ddots & \\
& & & -\frac{1}{c_{n-2}} & \frac{1}{c_{n-2}}+\frac{1}{c_{n-1}} & -\frac{1}{c_{n-1}} \\
& & & & -\frac{1}{c_{n-1}} & \frac{1}{c_{n-1}}+\frac{1}{c_{n}}
\end{array}\right] \\
& \boldsymbol{\Lambda}_{2}=\frac{c}{2 c}\left[\begin{array}{cccccc}
c_{1} & c_{1} & & & & \\
c_{1} & c_{1}+c_{2} & c_{2} & & & \\
& c_{2} & c_{2}+c_{3} & c_{3} & & \\
& & \ddots & \ddots & \ddots & \\
& & & c_{n-2} & c_{n-2}+c_{n-1} & c_{n-1} \\
& & & & c_{n-1} & c_{n-1}+c_{n}
\end{array}\right]
\end{aligned}
$$

$\boldsymbol{d}^{\mathrm{T}}=\left\{\begin{array}{llll}1 & 0 & \cdots & 0\end{array}\right\}_{n \times 1} ; \boldsymbol{u}=\left\{\begin{array}{lllll}u & u_{1} & u_{2} & \cdots & u_{n-1}\end{array}\right\} ; x$ and $z$ are the spatial coordinates (unit in $\mathrm{m}$ ); $t$ is time (unit in s); $u$ is the displacement (unit in $\mathrm{m}$ ); $c$ is the seismic wave velocity (unit in $\mathrm{m} / \mathrm{s}$ ); $u_{1}, u_{2}, \cdots, u_{n-1}$ are the auxiliary displacement variations (unit in $\mathrm{m}$ ) introduced in deducing the equation (1); $n$ is the order of equation, $c_{1}, c_{2}, \cdots, c_{n}$ represent phase velocities (unit in $\mathrm{m} / \mathrm{s}$ ) of seismic waves able to be accurately described by the arbitrarily wide-angle acoustic wave equation. The range of the values is $c_{i} \geq c(i=1,2, \cdots, n)$ and meets the relationship: $c / c_{i}=\cos \theta_{i}$. Guddati's study (2005) shows that the value of $\theta_{i}$ and $n$ jointly decide the dip range adaptable to migration of equation (1). When $n=2$, taking $c_{1}=c_{2}=c$, equation (1) is suitable for the migration of strata with a dip of about $60^{\circ}$, and taking $c_{1}=c, c_{2}=4 c$, equation (1) is suitable for the migration of strata with dip of about $80^{\circ}$; and when $n=3$, taking $c_{1}=c_{2}=c_{3}=c$, equation (1) is suitable for the migration of strata with dip of about $70^{\circ}$ and so on. For a detailed description of the relationship between the $n, c$ and $\theta_{i}$ consult Guddati (2005), as it is unnecessary to go into detail in this paper.

\subsection{High-order difference format of reverse-time travel of seismic waves}

The kernel using arbitrary wide-angle wave equations to implement prestack depth migration of P-wave data is how to solve the numerical solution of equation (1). Using a finite-difference algorithm, the paper deduced a high-order finite-difference format of the prestack reverse-time 
depth migration.

Based on the basic principle of the finite-difference algorithm, the difference discrete processing is carried out in space and time first. Setting the difference step-lengths in space $x, z$ and time $t$ as $\Delta x, \Delta z$ and $\Delta t$ respectively, and

$$
\left\{\begin{array}{l}
u_{i, j}^{k}=u(i \Delta x, j \Delta z, k \Delta t) \\
\boldsymbol{u}_{i, j}^{k}=\boldsymbol{u}(i \Delta x, j \Delta z, k \Delta t)
\end{array}\right.
$$

where $i, j$ and $k$ are order numbers of discrete points.

Difference discrete processing is carried out in the $z$ direction for equation (1) at point ( $i$, $j+1 / 2, k)$ and the following formula is obtained

$$
\frac{1}{\Delta z} \boldsymbol{d}\left(\frac{\partial u_{i, j+1}^{k}-\partial u_{i, j}^{k}}{\partial t}\right)-\frac{1}{c}\left(\boldsymbol{\Lambda}_{1}+\boldsymbol{\Lambda}_{2}\right) \frac{\partial^{2} u_{i, j+\frac{1}{2}}^{k}}{\partial t^{2}}+c \boldsymbol{\Lambda}_{2} \frac{\partial^{2} u_{i, j+\frac{1}{2}}^{k}}{\partial x^{2}}=0
$$

The following replacement is carried out for above formula:

$$
u_{i, j+1}^{k}=2 u_{i, j+\frac{1}{2}}^{k}-u_{i, j}^{k}
$$

Equation (3) becomes

$$
\frac{2}{\Delta z} \boldsymbol{d} \frac{\partial u_{i, j+\frac{1}{2}}^{k}}{\partial t}-\frac{1}{c}\left(\boldsymbol{\Lambda}_{1}+\boldsymbol{\Lambda}_{2}\right) \frac{\partial^{2} u_{i, j+\frac{1}{2}}^{k}}{\partial t^{2}}+c \frac{\partial^{2} u_{i, j+\frac{1}{2}}^{k}}{\partial x^{2}}=\frac{2}{\Delta z} \boldsymbol{d} \frac{\partial u_{i, j}^{k}}{\partial t}
$$

Using the difference quotient to replace derivative in both $x$ and $t$ directions and simplifying equation (5), we get the following formula:

$$
\begin{aligned}
& {\left[\left(\boldsymbol{\Lambda}_{1}+\boldsymbol{\Lambda}_{2}\right)+\frac{c \Delta t}{\Delta z} \boldsymbol{D}\right] u_{i, j+\frac{1}{2}}^{k-1}=-\frac{c \Delta t}{\Delta z} \boldsymbol{d}\left(u_{i, j}^{k+1}-u_{i, j}^{k-1}-u_{i, j+\frac{1}{2}}^{k+1}\right)-} \\
& \left(\boldsymbol{\Lambda}_{1}+\boldsymbol{\Lambda}_{2}\right)\left(u_{i, j+\frac{1}{2}}^{k+1}-2 u_{i, j+\frac{1}{2}}^{k}\right)+c^{2} \frac{\Delta t^{2}}{\Delta x^{2}} \boldsymbol{\Lambda}_{2} \sum_{m=-M}^{M} \alpha_{m} u_{i+m, j+\frac{1}{2}}^{k}
\end{aligned}
$$

where $\boldsymbol{D}=\boldsymbol{d} \boldsymbol{d}^{\mathrm{T}}, M$ is half of the difference order, $\alpha_{m}$ is difference coefficient (LIU et al, 1998).

Equation (6) is further sorted out and becomes the following formula:

$$
\boldsymbol{u}_{i, j+\frac{1}{2}}^{k}=-\frac{c \Delta t}{\Delta z} \boldsymbol{h}_{1}\left(u_{i, j}^{k+1}-u_{i, j}^{k-1}-u_{i, j+\frac{1}{2}}^{k+1}\right)-\boldsymbol{H}_{2}\left(\boldsymbol{u}_{i, j+\frac{1}{2}}^{k+1}-2 \boldsymbol{u}_{i, j+\frac{1}{2}}^{k}\right)+\frac{c^{2} \Delta t^{2}}{\Delta x^{2}} \boldsymbol{H}_{3} \sum_{m=-M}^{M} \alpha_{m} \boldsymbol{u}_{i+m, j+\frac{1}{2}}^{k}
$$

Where $\boldsymbol{h}_{1}=\boldsymbol{L} d, \boldsymbol{H}_{2}=\boldsymbol{L}\left(\boldsymbol{\Lambda}_{1}+\boldsymbol{\Lambda}_{2}\right), \boldsymbol{H}_{3}=\boldsymbol{L} \boldsymbol{\Lambda}_{2}, \quad \boldsymbol{L}=\left(\boldsymbol{\Lambda}_{1}+\boldsymbol{\Lambda}_{2}+\frac{c \Delta t}{\Delta z}\right)^{-1}$.

Extensive numerical studies show that the computation of equation (7) is conditionally stable for

$$
c \Delta t \leq \min (\Delta x, \Delta z)
$$

We take equation (8) as the stability condition of reverse-time finite different scheme (7). A detailed process for deducing stability conditions is outside the scope of this paper. Simultaneously solve equations (7) and (4) can realize reverse-time travel of P-wave data.

In practical processing, the wave field should be computed from shallow to deep in $z$ direc- 
tion. Assuming the value $u_{i, j}^{j-1}$ is known, the vector $\boldsymbol{u}_{i, j+\frac{1}{2}}^{k-1}$ is first computed by equation (7), then, $u_{i, j+1}^{k-1}$ is computed by equation (4), and the recursion in this order is carried out until the evaluation of displacements of discrete points in total depth is finished.

\subsection{Boundary conditions}

Since equation (1) is a one-way wave equation, the up-going wave is not produced in the process of reverse-time travel of seismic waves, so equations (7) and (4) can be used directly to compute the wave field in the bottom boundary and no special algorithm is needed. Concerning the top boundary, we only need to add a grid layer above the receiver points and use a simple rigid boundary $(u=0)$, which can achieve the reflection-free object on the boundary. As for the left and right boundaries, this paper uses the weighted direction correction and interpolation-predicting boundary condition (LUO et al, 1999) to suppress pseudo-reflections from the truncation boundary. More accurate boundary conditions for reverse-time migration of arbitrarily wide-angle wave equations are now in study.

\subsection{Imaging conditions}

Imaging conditions is one of the main content in prestack reverse-time migration, via a simple, one-interface acoustic model, Chattopadhyay et al (2008) studied the migration result of excitation-time imaging conditions, cross-correlation imaging conditions and amplitude-ratio imaging conditions, which imply that in the same computational parameters the amplitude-ratio imaging conditions can provide the best resolution. But in practical computation, applying cross-correlation imaging conditions and amplitude-ratio imaging conditions need to do a forward modeling of given interval velocity model, which increase the calculation amount to two times of reverse-time propagation, moreover the application of these two imaging conditions also needs extensive computer time, when dealing with the huge velocity model problem, the time used to applying the imaging condition can be even longer than that used in migration. Taking the processing efficiency into account; the paper uses excitation-time imaging conditions to implement the reverse-time migration of arbitrarily wide-angle wave equations.

The computation of excitation-time imaging conditions can be achieved by solving the eikonal equation that is as follows in 2-D isotropic medium:

$$
\left(\frac{\partial t}{\partial x}\right)^{2}+\left(\frac{\partial t}{\partial z}\right)^{2}=\frac{1}{c^{2}}
$$

The solutions of equation (9) have been systematically studied by many researchers. This paper uses an expanding wave face algorithm based on the up-wind format (HE and ZHANG, 2006; Vidale, 1990) to get the imaging conditions needed for prestack reverse-time depth migration of arbitrarily wide-angle acoustic wave equation.

\section{Pulse response of prestack reverse-time depth migration of arbi- trary wide-angle acoustic wave equation}

In order to test the precision of the migration operator given by equations (7) and (4), we select different $n$ and $c_{i}$ to compute the pulse response. Figure 1 shows the computed results. The location of the pulse is at $x=1500 \mathrm{~m}$ and $t=0.4 \mathrm{~s}$, the dominant frequency of the pulse is $30 \mathrm{~Hz}$, and the velocity in the homogeneous medium is $3000 \mathrm{~m} / \mathrm{s}$. The ideal response curve is shown as a white line in the figure, the half-circle-like events pointed out by the arrows in the upper part of 
each figure are errors brought by deducing equation (1), which is characterized by weak energy and a minor influence on migrated results and the suppression method of this event is beyond the scope of this paper.

The figure shows that the maximal dip angle accurately imaged by arbitrary wide-angle wave equation is $60^{\circ}$ with $n=2$ and $c_{1}=c_{2}=c, 80^{\circ}$ with $n=2$ and $c_{1}=c, c_{2}=4 c$, and $70^{\circ}$ with $n=3$ and $c_{1}=c_{2}=c_{3}=c$, which proves the analytic results presented by Guddati (2005), that is, $n$ and $c_{i}$ jointly decide the dip range of migration applicable for the approach. When implementing the algorithm in the computer, if $n$ is unchanged, the total computational efforts are unchanged no matter what value is used for $c_{i}$. The computational time will double or redouble when $n$ increased. So, using the parameters in Figure 1b, one can achieve a satisfactory migration result when the angle of bedding is less than $80^{\circ}$ in practical data processing. The selection of $n$ and $c_{i}$ in the following processing is the same as shown in Figure $1 b$.

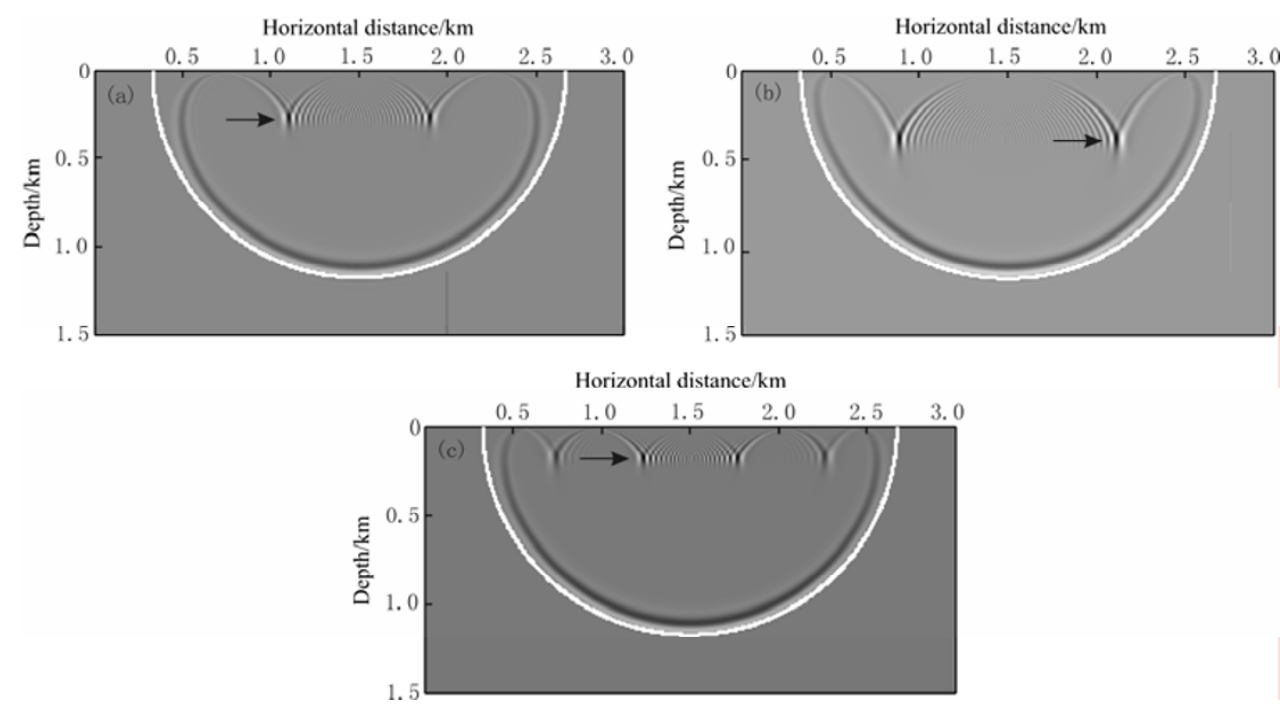

Figure 1 The prestack reverse-time impulse response of arbitrary wide-angle wave equations with different parameters

(a) $n=2, c_{1}=c_{2}=c$; (b) $n=2, c_{1}=c, c_{2}=4$ c; (c) $n=3, c_{1}=c_{2}=c_{3}=c$

\section{Migration tests of theoretical models}

\subsection{Model 1}

Model 1 is shown in Figure 2a. There are five reflectors in the homogeneous isotropic medium, with dips of $75^{\circ}, 65^{\circ}, 45^{\circ}, 25^{\circ}$ and $0^{\circ}$, respectively. The velocity of homogeneous isotropic medium is $4000 \mathrm{~m} / \mathrm{s}$. Figure $2 \mathrm{~b}$ shows poststack synthetic records of the model with a group interval of $10 \mathrm{~m}$. Figure $2 \mathrm{c}$ shows the results of poststack depth migration using the algorithm provided by this paper, in which the migrated results are totally coincident with the practice in the bed dips, which illustrates that this method can be used in poststack processing and is also applicable for steep dip migration. The reason that some indistinct event are still exist in the areas where there is no interface lies in that theoretically, the reflection from a subsurface diffractor can be received at any position in earth surface, but the acquisition and migration of seismic wave can only carried out in a limited area, so this finite migration aperture result in some indistinct event in no-interface areas which is regarded as the migration noise. 


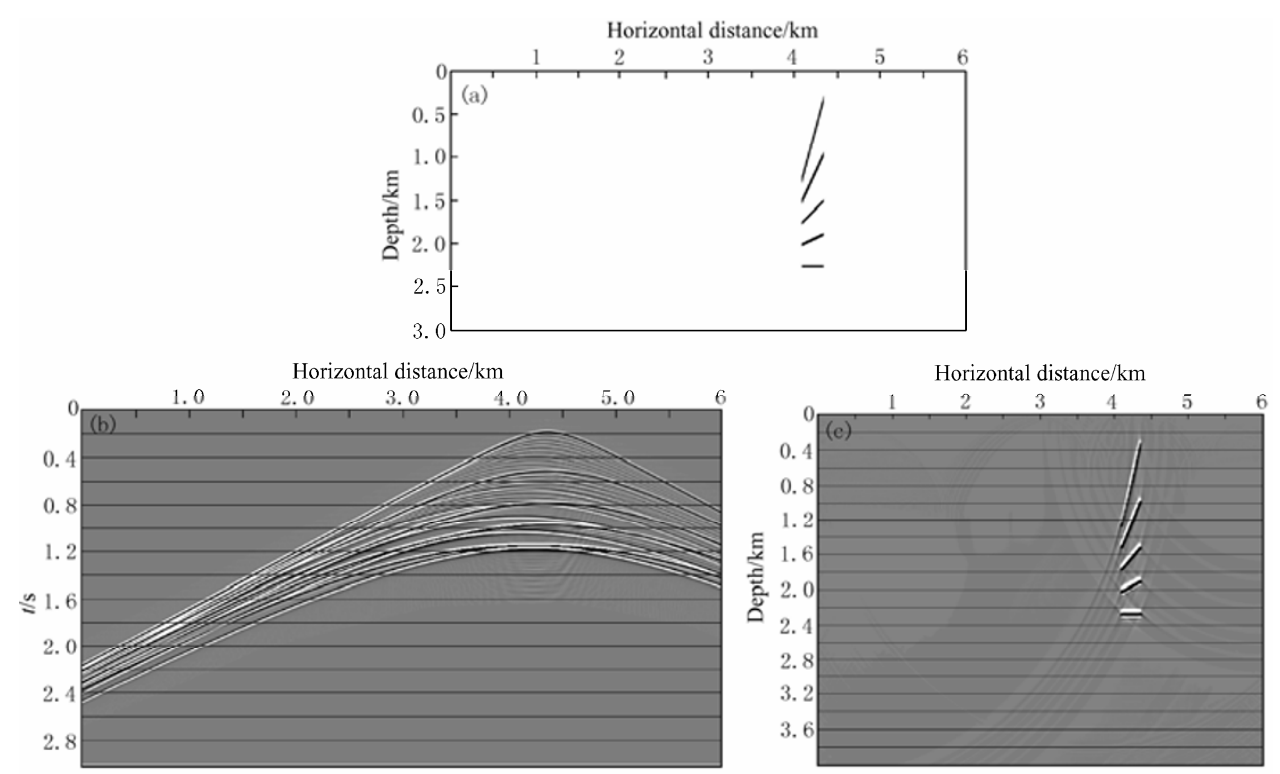

Figure 2 Theoretical model, synthetic seismogram and migration result of arbitrary wide-angle wave equations

(a) Layout of the numerical experiment; (b) The synthetic zero-offset seismogram of Figure 2a; (c) Migration result of Figure $2 b$

\subsection{Model 2}

Model 2 is a horizontal layered medium with two layers, the velocities of which are 3000 $\mathrm{m} / \mathrm{s}$ and $3500 \mathrm{~m} / \mathrm{s}$ respectively. The depth of the elastic interface of two layers is $300 \mathrm{~m}$. Figure $3 \mathrm{a}$ is a synthetic seismic record of a single shot; Figure $3 b$ and $3 c$ are the results of the reverse-time migration using an arbitrary wide-angle acoustic wave equation and the two-way acoustic wave equation, respectively. Both can better reflect the features of the reflection interface. Low-frequency noise with stronger energy appears above the reflection interface in Figure $3 \mathrm{c}$ (see the arrow in figure). The snapshot of reverse-time propagation (Figure 3d) shows that the noise is mainly produced by interval reflections of down-going wavefield (the arrow point to reflections of interface). This noise will deteriorate the migration results of overlying stratum, especially in the region close to the source. It is the main shortcoming of two-way wave equation reverse-time migration technique which restricts the industrial application of this technique. But for arbitrarily wide-angle equations, it can be seen from the snapshot (Figure 3e) that the strata interface does not produce reflections for the down-going wavefield, which eliminates the influence of interval reflections on the migrated results.

\subsection{Model 3}

As a practical benchmark test, the Marmousi model is migrated with the proposed method. The velocity model and reflector position can be seen in Figure 4a. The synthetic seismograms are obtained by a finite different scheme with a compressional source of $35 \mathrm{~Hz}$ dominant frequency. There are total 63 shots synthetic data are used to do migration. All synthetic shots have the same receiver position and were recorded by 766 channels with $10 \mathrm{~m}$ group interval, $0.4 \mathrm{~ms}$ sample interval and $4 \mathrm{~s}$ recording length. The shot interval is $120 \mathrm{~m}$, and the coordinate of the first and last shot is $(0,0)$ and $(7560,0)$ respectively. Figure $4 \mathrm{~b}$ shows one of the synthetic seismogram when the shot coordinate is $(1200,0)$. Figure $4 \mathrm{c}$ is the stacked section of migrated results. In the migra- 

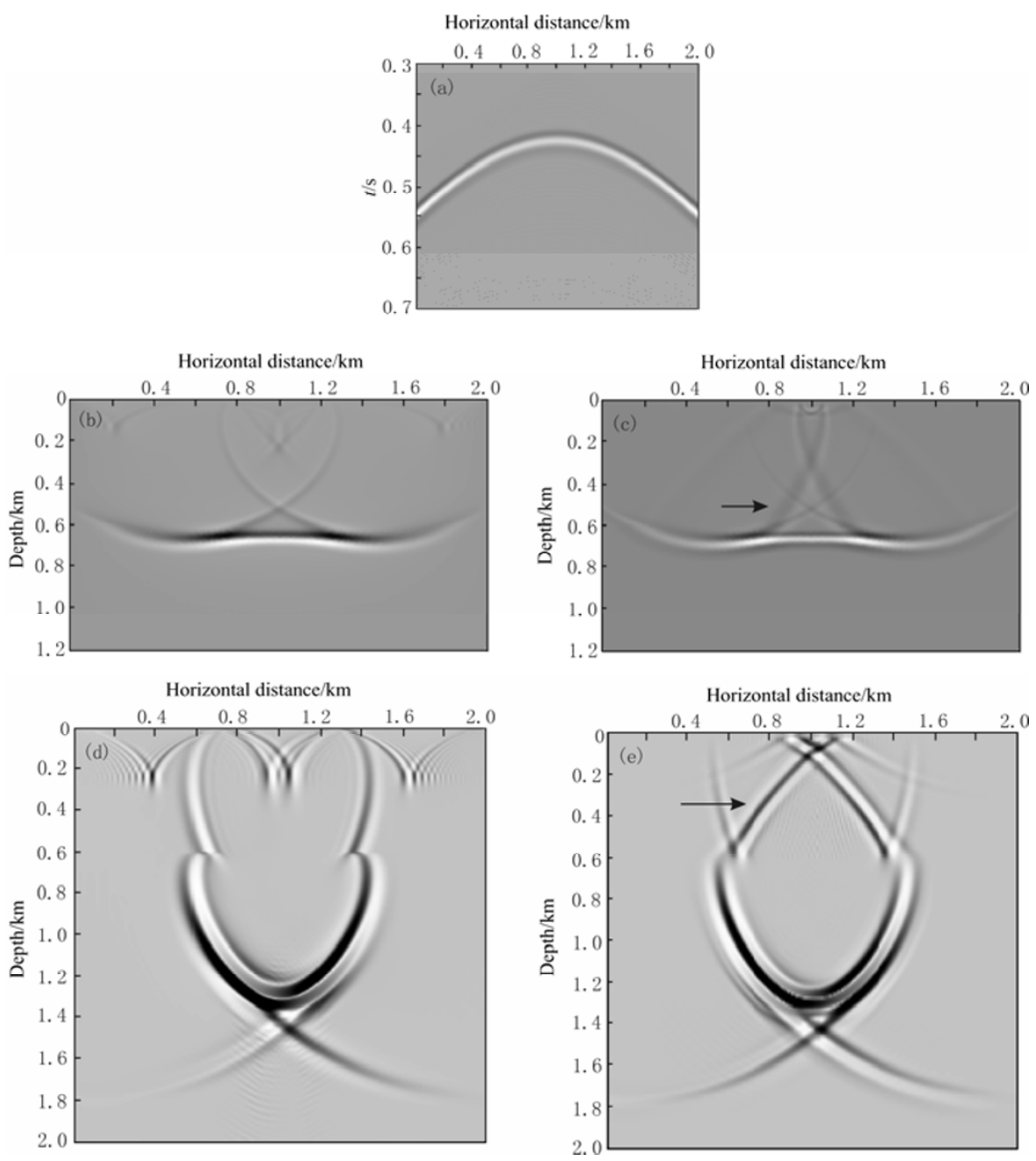

Figure 3 Synthetic seismogram of a two layer horizontal model, migration result and snapshot using different wave equations

(a) Synthetic seismogram of two-layer horizontal model; (b) Migration result of arbitrary wide-angle wave equations; (c) Migration result of two-way wave equations; (d) Snapshot of arbitrary wide-angle wave equations reverse-time propagation; (e) Snapshot of two-way wave equations reverse-time propagation

tion result, three major faults, high velocity stratum and all detail of Marmousi are displayed in the migration section. Because the finite difference scheme proposed in the paper subdivide the geologic model with rectangular grid, some step-shaped interfaces are occurred after gridding the velocity model even the original interface is smooth. Decreasing the grid size can remove above error effectively, but the small grid size means the increasing of the grid number and computation time, so in practical processing, the grid size should be chosen according to both geological structure and the accuracy requirement of migration to satisfy the migration efficiency and accuracy.

\section{Conclusions}

The arbitrary wide-angle wave equation is deduced by reasonable simplification of two-way wave equation. It can describe the down-going wavefield accurately and can to be used to solve 

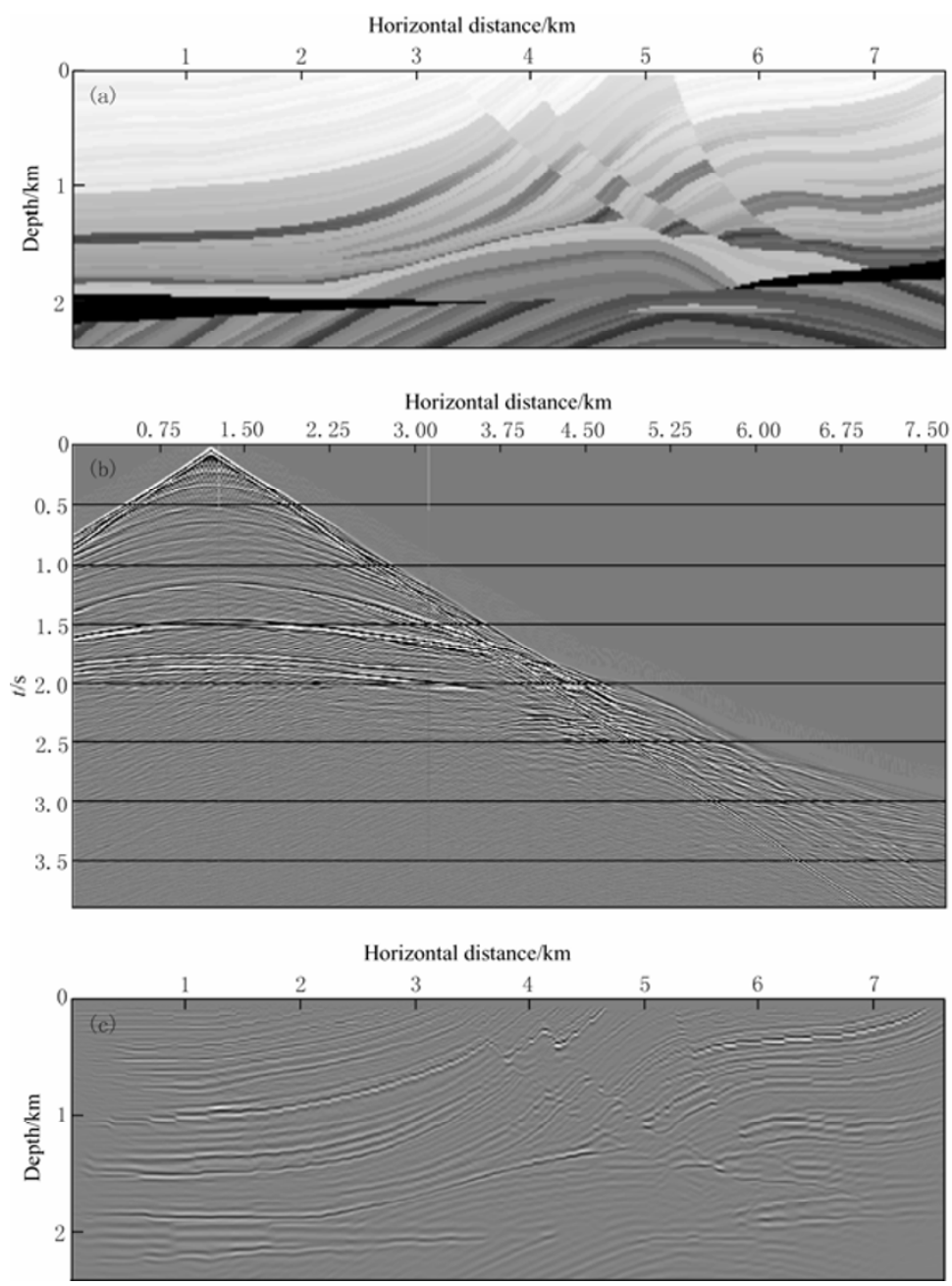

Figure 4 Theoretical model, synthetic seismogram and migration results

(a) Marmousi velocity model; (b) One of the synthetic seismogram of Figure 4a; (c) Stack profile of 63 shots migration results

the issue of prestack reverse-time depth migration.

The prestack reverse-time migration algorithm based on arbitrarily wide-angle wave equation can remove the unwanted interval reflections and decrease the migration noise.

The prestack reverse-time depth migration algorithm based on arbitrarily wide-angle wave equation also has the advantages of high amplitude fidelity and applicable to implement the migration of dip angle structure.

The paper studied the issue of the prestack reverse-time depth migration algorithm based on 2-D arbitrary wide-angle wave equation, but the relevant ideas and algorithm can be conveniently used in 3-D data processing.

\section{References}

Berfenger J P. 1994. A perfectly matched layer for the absorption of electromagnetics waves [J]. Journal Computation Physics, 114(2): $185-200$.

Chattopadhyay S and McMechan G A. 2008. Imaging conditions for prestack reverse-time migration [J]. Geophysics, 73(3): S81-S89. 
Claerbout J F. 1985. Imaging the Earths Interior [M]. Osney Mead: Blackwell Scientific Publications: 1-412.

Gazdag J. 1978. Wave equation migration with the phase shift method [J]. Geophysics, 43(10): 1342-1351.

Guddati M N and Heidari A H. 2005. Migratrion with arbitrarily wide-angle wave equations [J]. Geophysics, 70(3): S61-S70.

Guitton A, Kaelin B, Biondi B. 2007. Least-squares attenuation of reverse-time-migration artifacts [J]. Geophysics, 72(1): S19-S23.

HE Bing-shou and ZHANG Hui-xing. 2006. Vector prestack depth migration of multi-component wavefield [J]. Oil Geophysical Prospecting, 41(4): 369-374 (in Chinese).

LIU Yang, LI Cheng-chu, MOU Yong-guang. 1998. Finite-difference numerical modeling of any even-order accuracy [J]. Oil Geophysical Prospecting, 33(1): 1-10 (in Chinese).

Loewenthal D and Mulfti I R. 1983. Reverse-time migration in spatial frequency domain [J]. Geophysics, 46(3): 627-635.

LUO Da-qing, SONG Wei, WU Lü. 1999. 3-D wave equation prestack numerical simulation using the absorbing boundary condition that needs weighting-directional correction and interpolation prediction [J]. Oil Geophysical Prospecting, 34(3): 275-284 (in Chinese).

McMechan G A. 1983. Migration by extrapolation of time-dependent boundary values [J]. Geophys Prosp, 31(2): 413-420.

Robin P F, Paul J F, Phil K, et al. 2006. Suppressing unwanted internal reflections in prestack reverse-time migration [J]. Geophysics, 71(6): E79-E82.

Symes W W. 2007..Reverse-time migration with optimal checkpointing [J]. Geophysics, 72(5): SM213-SM221.

Vidale J. 1990. Finite difference calculation of traveletimes in three dimension [J]. Geophysics, 55(5): 521-526.

Whitemore N D.1983. Iterative depth migration by backward time propagation [G]//The 53th Annual International Meeting. SEG Expanded Abstracts: $827-830$.

Yoon K and Marfurt K J. 2006. Reverse-time migration using the pointing vector [J]. Exploration Geophysics, 59(1): 102-107. 